\title{
Pregnancy outcomes, early separation and psychiatric relapse experienced by South African women with peripartum psychosis: A descriptive exploration
}

\section{Juané Voges ( $\nabla$ jvoges@sun.ac.za )}

Stellenbosch University Faculty of Medicine and Health Sciences https://orcid.org/0000-0003-18398371

\author{
Astrid Berg \\ University of Cape Town Department of Psychiatry and Mental Health \\ Dana JH Niehaus \\ Stellenbosch University Faculty of Medicine and Health Sciences
}

\section{Research article}

Keywords: Peripartum psychosis, pregnancy outcome, mother-infant separation, prenatal substance use, postpartum relapse

Posted Date: December 17th, 2019

DOI: https://doi.org/10.21203/rs.2.18975/v1

License: (c) (1) This work is licensed under a Creative Commons Attribution 4.0 International License.

Read Full License 


\section{Abstract}

Background: The peripartum period is associated with increased incidence of severe mental illness (SMI), with a greater occurrence of psychotic symptoms and psychiatric admissions. There has been little research on South African mothers with peripartum psychosis to examine what factors contribute to their experiences of pregnancy and the postpartum period. This study aimed to explore the relevant sociodemographic factors and pregnancy outcomes in order to determine focus areas for intervention.

Methods: Using a prospective, descriptive design, we conducted a detailed interview and supplementary information was collected by review of clinical records. The study examined the accounts of thirty-two mothers who experienced peripartum psychosis between 6 and 18 months postpartum. Areas of focus included participants' current circumstances, their pregnancy, birth and postpartum experiences.

Results: The majority of mothers were diagnosed with bipolar disorder and all but one have had one psychiatric admission. Pregnancies were predominantly unplanned (87.5\%) and half of participants reported substance use during pregnancy. Medical or psychiatric problems during pregnancy occurred in $68.8 \%$ of pregnancy and $71.9 \%$ of mothers reported complications with delivery. Common psychosocial stressors included trauma, lack of partner support and financial hardship. Early separation occurred in $56.3 \%$ of dyads, mostly due to psychiatric admission.

Conclusion: Our study describes psychiatric, medical and psycho-social risk factors affecting mothers who experienced peripartum psychosis. We discuss the implications of the findings for a developing country where there exists a lack of integrated maternal and infant mental health services. The results highlight the need for close obstetric and psychiatric follow-up for women with serious mental illness, with particular attention to screening for substance use and psychosocial stressors.

\section{Introduction}

Pregnancy and the postpartum period is often experienced as a time of great joy and excitement for many women. However, this phase is also associated with vulnerability to mental illness, risk of relapse of pre-existing mental illness, and the development of new psychiatric symptoms. Perinatal mental illness can be defined as psychiatric conditions that manifest during pregnancy and up to one year after delivery (1).

During pregnancy and postpartum, there is an increased incidence of severe mental disorders (SMI), which may include disorders such as schizophrenia, affective psychosis and bipolar disorder. During the post-partum period, there is a dramatic increase in the frequency of psychotic symptoms and psychiatric admissions $(2,3)$. These symptoms may be manifestations of a new illness that develops during the postpartum period, or a relapse of a pre-existing illness. The presence of serious mental illness (SMI) in the perinatal period can have potentially devastating consequences for the mother, infant and family system and may adversely affect the mother's capacity to parent $(4,5)$. 
Factors that contribute to the increased incidence of relapse and new-onset mental disorders during the perinatal period include changes in medication, sleep deprivation, hormonal variation, as well as the physical and psychological demands of pregnancy and infant care $(2,6)$.

Examining the risk factors for relapse of SMI during pregnancy, Taylor and colleagues (7) found a relapse rate of $12 \%$ for women with affective psychoses and $24 \%$ for women with non-affective psychoses. Risk factors for relapse of SMI included non-affective psychosis, number of recent psychiatric admissions, recent self-harm, substance abuse, smoking and non-white ethnicity. Extending their work to postpartum women, Taylor and colleagues (8) found that $28.3 \%$ of women with SMI relapsed within the first three months postpartum. Relapses were associated with smoking, as well as with the recency and frequency of relapses prior to pregnancy.

The presence of serious psychiatric disorders, in particular psychotic disorders, places the mother and unborn infant at greater risk for adverse obstetric and neonatal outcomes (9). The risk of stillbirth or delivering an infant with a low birthweight doubles if the psychotic episode occurs during pregnancy (10). Expectant mothers who have a pre-existing psychotic disorder may be at greater risk for obstetric complications than those mothers who develop postpartum psychotic disorders (11). Pregnancy outcomes of mothers with affective psychoses, such as bipolar disorder, carry double the risk for a preterm birth, low birthweight or small for gestational age babies (12).

Substance use during pregnancy also contributes to the risk for adverse obstetric and child outcomes (13). Zhao and colleagues (14) reported that poorer birth outcomes among women with mental illness were more likely when the mother used substances during pregnancy. The high rates of substance use among South African pregnant women has been recognised by two surveys of women attending Midwife Obstetric Units in Cape Town where alcohol consumption and smoking were found to be especially prevalent $(15,16)$.

Postpartum psychosis in particular is considered to be one of the most severe psychiatric conditions with onset in the postpartum period (17). The phenomenon of postpartum psychosis refers to the sudden onset of manic or psychotic symptoms in the first 6 weeks after delivery (18). The clinical symptoms typically associated with postpartum psychosis include a range of psychotic phenomena (delusions and hallucinations) that often relates to the infant; affective symptoms (elation and depression); and disturbances of consciousness (which may be in the form of confusion, bewilderment, perplexity) $(2,19)$. Sudden changes in the clinical presentation with significant fluctuations in the intensity of symptoms are common and have been described as the "kaleidoscopic picture of postpartum psychosis" (18, p1182).

In general, postpartum psychosis occurs relatively rarely. In a systematic review, VanderKruik and colleagues (20) reported incidence rates ranging between 0.89 and 2.6 per 1000 women, but noted variations in study methodology, definition and assessment of postpartum psychosis. Although uncommon, postpartum psychosis may place the mother at increased risk for suicide and infanticide $(2,18)$. 
There is compelling evidence that women with a history of bipolar disorder are at a significantly increased risk of developing postpartum psychosis $(3,21)$, which places them at a higher risk for psychiatric admission and separation from their infants.

Although women with schizophrenia and bipolar disorder have somewhat lower fertility rates than women in the general population, the majority of these women are parents (2). However, Miller and Finnerty (22) reported a greater likelihood that the pregnancies of women with schizophrenia would be unplanned and unwanted when compared to women without the disease.

According to Cès and colleagues (23), mothers suffering from psychotic disorders are at greater risk of being separated from their infants. Separations between mother and infant frequently occur when mothers experience SMI and in particular postpartum psychosis and require inpatient psychiatric treatment.

During the first half of the twentieth century, separation of mothers with postpartum psychotic disorders from their babies were considered to be advantageous for both parties (24). The emergence of social psychiatry, changes in psychiatric treatment, as well as research by Bowlby (25) and Spitz (26) on the adverse effects of early separation from attachment figures signaled a questioning of this approach (24). More recent studies have further provided support for the notion that early separation between infants and mothers may have adverse consequences. Separations between the mother and infant occurring during the infant's first year pose a risk to the development of a secure attachment relationship (27). Furthermore, Howard and colleagues (28) found that mother-infant separation within the first two years of life was associated with child negativity at age three and aggression at ages three and five. In recognition of the benefits of maintaining proximity of mothers and infants during the postpartum period, psychiatric mother-baby units (MBUs) emerged in several developed countries with favorable outcomes for postpartum mothers (29).

The first joint admissions of mothers with schizophrenia and their babies occurred in 1959. Baker and colleagues (30) demonstrated that mothers admitted with their babies had shorter periods of admission, were more likely to care for their babies upon discharge and had lower rates of relapse than mothers treated in an admission ward without their babies. This practice has now become more widespread and is recommended by the National Institute for Health and Clinical Excellence (31). Despite the benefits of joint admissions, mothers with psychotic disorders may still be separated from their infants as reported by Cès and colleagues (23) who analyzed joint admissions of mothers with psychotic disorders and their infants. The authors linked early separation to the placement of the mother in an institution during childhood, being single, early hospitalization of the baby and maternal psychiatric decomposition during pregnancy.

Du Toit and colleagues identified several risk factors contributing to unplanned pregnancies in a sample of South African women with psychiatric illness (32). These include being of younger age, two or more pregnancies in the past, being of mixed ancestry, African or Indian ethnicity, being unmarried, below tertiary level of education, being unemployed, low socio-economic status, substance abuse, previous 
psychiatric admissions and previous suicide attempts. The presence of maternal mental illness in combination with unplanned pregnancy significantly increases the vulnerability of these mothers to adverse pregnancy outcomes $(32,33)$.

Mothers utilizing mental health services may experience more significant challenges in their roles as parents, which may be of particular significance during infancy, when there is greater dependence on the parent (34). Children in care of mothers with psychiatric illnesses may be more vulnerable to developing insecure or disorganized attachment, particularly when maternal psychopathology is severe and prolonged, and when other risk factors such as parental trauma are present (35). The presence of both maternal psychiatric symptoms and separations may adversely affect the quality of the mother-child relationship, as well as parental sensitivity and capacity (36).

Specific features of psychotic disorders, such as withdrawal, delusional thinking, disorganized behavior and reduced responsiveness, may separately and in combination hamper the mother's ability to provide a consistent, attuned presence for her infant and may compromise the development of a secure attachment, especially if early separation occurs $(4,28)$.

Nurturing care is defined by the World Health Organization (WHO) as "giving young children opportunities for early learning, through interactions that are responsive and emotionally supportive" (37, p2) and focusses on the importance of the period from pregnancy to age 3 . Several components make up nurturing care, including: behaviors, attitudes, knowledge regarding caregiving, stimulation,

responsiveness and safety (38). Mothers with peripartum psychosis may require additional support in the provision of nurturing care in order to provide adequate opportunities for learning and development for their infants.

In the South African context of widespread socio-economic risks and limited resources, it is imperative to identify vulnerable dyads for early intervention. To our knowledge, there has not been a study among South African women with experiences of peripartum psychosis. The present study aims to provide a description of the socio-demographic factors and pregnancy outcomes for this group of women to determine areas of focus for intervention. By gaining a better understanding of the potential difficulties that mothers with this severe form of peripartum psychiatric illness face, dyads who may be at particular risk for adverse maternal and infant outcomes may be identified for further treatment.

\section{Methods}

\section{Study design}

This prospective study followed a quantitative and descriptive design. Thirty-two mothers who had experienced peripartum psychosis were recruited from three public hospitals in South Africa between 2016 and 2019.

\section{Study setting}


The recruitment sites included Stikland Hospital, a psychiatric facility providing care for adult in- and outpatients; Tygerberg Hospital, a tertiary and academic hospital; and Karl Bremer Hospital, a district hospital in the Western Cape. These hospitals serve a South African community with predominantly low to middle socio-economic circumstances.

\section{Participants}

We used purposive sampling to specifically recruit mothers who had experienced peripartum psychosis. Mothers were eligible for the study if they gave birth to a healthy infant in the last 18 months and if they experienced psychotic symptoms during their pregnancy and/or within six months postpartum. In cases where mothers exhibited moderate to severe psychotic symptoms at the time of assessment, inclusion was delayed until the symptoms were resolved. Inclusion in the larger study required mothers to reside with their infants and as such, mothers were excluded from the sample if the infants were no longer in their care.

\section{Procedure}

Participants were referred to the study and invited for a research visit at Stikland Hospital between 6 and 18 months postpartum. Participants were determined to have capacity to provide consent for the study if they understood the information provided and could appreciate the implications of their participation

(39). Participants were informed of the nature and procedure of the study, all study-related questions were answered, and informed consent was obtained. Thereafter, demographic information was collected through a detailed interview and information was supplemented by review of clinical records. The positive subscale of the Structured Clinical Interview for the Positive and Negative Symptoms Scale (SClPANSS) was completed to asses for current psychotic symptoms.

Peripartum psychosis was identified by their multi-disciplinary treatment teams who verified the presence of psychotic symptoms in the peripartum period and the primary investigator reviewed clinical records for additional information. At the time of the research visit, the stability of symptoms was evaluated from clinical records and a clinical interview with all participants. Two individuals experienced moderate to severe psychotic symptoms at the time of initial interview as assessed by the SCI-PANSS and were offered a later date to complete the interview when their clinical symptoms had stabilized.

\section{Instruments}

\section{Demographic interview}

A demographic interview explored aspects of the mothers' accounts of their current circumstances, pregnancy, birth and postpartum experience. The demographic information collected included age, level of education, marital status, separation periods between mother and child, medical and psychiatric history obtained from the direct interview. Where possible, information was verified through an audit of the clinical files.

\section{Positive and Negative Symptoms Scale (PANSS)}


Current psychotic symptoms was assessed by means of the Structured Clinical Interview for the Positive and Negative Symptoms Scale (SCI-PANSS). The Positive and Negative Symptoms Scale (PANSS) is a widely-used tool for evaluating symptoms in schizophrenia that has been found to have adequate internal consistency and reliability (40) and has been utilized to assess psychopathology symptoms in South African populations (41). The positive subscales of the interview were completed, and each item assessed on a 7-point Likert scale ranging from 1 (absent) to 7 (extreme). A cut-off score of 4 on any of the positive subscale items was used. Scores of 4 and above indicate the presence of at least moderate psychotic symptoms. Participants who scored above 3 on any item were provided with support to access psychiatric review and provided with a later date to complete the assessment.

\section{Data analysis}

Data were analyzed with SPSS for Windows, version 25. The distribution and descriptive statistics are provided for demographic and clinical variables.

\section{Ethical considerations}

Permission to conduct the study and ethical approval for the study was obtained in accordance to the provisions and approval of the Health Research Ethics Committee of Stellenbosch University (S16/01/014). Consent for participation in the study was obtained from the participants. Participation in the study was voluntary and the participants could withdraw at any point without coercion or any negative consequences. Participants were provided with transport, received refreshments during the procedure and were given payment for their participation in the study.

\section{Results}

\section{Demographic information}

Thirty-two mothers were eligible for the study over the period of four years and their demographic information is provided in Table 1. Mothers ranged in age between 21 and 44 years (mean $=29.7$ ) and had between $1(37.5 \%)$ and 4 children (9.4\%). The majority of mothers achieved a secondary level of education (81.3\%). A third of the mothers were married (34.4\%), with the remainder categorized as in a relationship (37.5\%) or single (28.1\%). Less than a third were employed $(28.1 \%)$, and a similar number $(28.1 \%)$ were receiving a disability grant. The majority of mothers $(71.9 \%)$ had an annual household income of less than R100 000 (equivalent to approximately $\$ 6920$ ). 
Table 1

Demographic variables $(n=32$, unless otherwise noted $)$

\begin{tabular}{|ll|}
\hline Variable & Statistic \\
\hline Mean age (years) & $29.7(\mathrm{sd}=5.4)$ \\
\hline Level of education & $\%(\mathrm{n})$ \\
\hline Primary school & $6.3(2)$ \\
\hline Secondary school & $81.3(26)$ \\
\hline Tertiary education & $12.5(4)$ \\
\hline Relationship status & \\
\hline Single & $28.2(9)$ \\
\hline In a relationship & $37.5(12)$ \\
\hline Married & $34.4(11)$ \\
\hline Employment status & \\
\hline Unemployed & $40.6(13)$ \\
\hline Receiving a disability grant & $28.1(9)$ \\
\hline Employed & $28.1(9)$ \\
\hline Scholar & $3.1(1)$ \\
\hline Socio-economic status as indicated by annual household income & $9.4(3)$ \\
\hline Receiving a social grant & $71.9(23)$ \\
\hline <R100 000 & $9.4(3)$ \\
\hline <R350 000 & $3.1(1)$ \\
\hline OR350 000 private medical aid & $6.3(2)$ \\
\hline
\end{tabular}

\section{Maternal medical and psychiatric information}

Fourteen mothers were diagnosed with bipolar disorder (43.8\%), 9 were diagnosed with schizophrenia (28.1\%), and 3 mothers either had a diagnosis of major depressive disorder with psychosis or schizoaffective disorder (9.38\%). The distribution of diagnoses and comorbidities are provided in Table 2. All but one of the mothers $(96.9 \%)$ have had at least one psychiatric admission, with over half $(56.3 \%)$ being admitted prior to their pregnancy, $28.1 \%$ admitted during their pregnancy and just over half (53.1\%) admitted subsequent to their pregnancy. One mother was admitted during pregnancy and discharged 8 weeks postpartum. Of the further 16 mothers who were admitted during the postpartum period, 6 (37.5\%) 
were admitted within 8 weeks of delivery. Total scores for the positive subscale of the PANSS at the time of the assessments ranged between 7 and 14, with a median of 9 , and a mean of 9.3 (standard deviation, $s d=1.9$ ). The median item score was 2 and the maximum score for any item was 3 , which indicates the presence of mild symptoms that do not generally interfere with functioning.

Table 2

Primary psychiatric diagnosis and comorbidity

\begin{tabular}{|ll|}
\hline Primary psychiatric diagnosis & $\%(\mathbf{n}=\mathbf{3 2})$ \\
\hline Bipolar disorder & $43.8(14)$ \\
\hline Schizophrenia & $28.1(9)$ \\
\hline Schizoaffective disorder & $9.4(3)$ \\
\hline MDD with psychosis & $9.4(3)$ \\
\hline Schizophreniform disorder & $6.3(2)$ \\
\hline Substance-induced psychotic disorder & $3.1(1)$ \\
\hline Comorbid diagnosis categories & \\
\hline Substance use disorder & $15.6(5)$ \\
\hline Personality disorder/traits & $6.3(2)$ \\
\hline Intellectual disability & $6.3(2)$ \\
\hline Conversion disorder & $3.1(1)$ \\
\hline Table 3. Use of substances during pregnancy & \\
\hline Substance & $\%(n=32)$ \\
\hline Smoking & $40.6(13)$ \\
\hline Alcohol & $21.9(7)$ \\
\hline Illicit drugs & $12.5(4)$ \\
\hline
\end{tabular}

\section{Pregnancy and delivery}

More than four fifths of the pregnancies were unplanned (87.5\%), but only $6(18.8 \%)$ were unwanted. The frequencies of substances used during pregnancy are provided in Table 3. Substance use during pregnancy was reported by half $(50.0 \%)$ of mothers, with cigarette smoking being most prevalent $(40.6 \%)$ and alcohol use present in $21.9 \%$ of pregnancies. Six mothers (18.8\%) used more than one substance during pregnancy. Post-pregnancy, some mothers abstained from using substances, with only 12 mothers $(37.5 \%)$ reporting substance use at the time of the interview. The majority of mothers $(68.8 \%)$ reported 
either medical or psychiatric problems during their pregnancy and half (50.0\%) reported psychotic symptoms during their pregnancy.

Births took place between 30 weeks and 43 weeks gestation, with a mean gestational age of 38 weeks. Fourteen mothers delivered via vertex delivery (43.8\%) and there were twelve emergency caesarean sections (37.5\%). A high number of mothers (71.9\%) self-reported complications during the delivery, including "pre-eclampsia" ( $n=2 ; 8.7 \%)$, "cervix not dilating" $(n=3 ; 13.0 \%)$, "tearing" $(n=8 ; 34.8 \%)$, and "infection" ( $n=2 ; 8.79 \%)$. Mothers reported that infants experienced "distress" $(n=2 ; 8.7 \%)$, "irregular heartrates" ( $n=3 ; 13.0 \%)$, "were placed in an incubator" ( $n=5 ; 21.7 \%)$, "were cyanotic or blue" $(n=2$; $8.7 \%)$, "needed oxygen" ( $n=3 ; 13.0 \%)$ and had "a low birth weight" $(n=4 ; 17.4 \%)$.

\section{Post-partum: Stressful life events}

Over half of the mothers (68.5\%) reported a history of trauma, with either physical or sexual abuse identified in 12 mothers (37.5\%). The most frequently reported psychosocial stressor was lack of partner support $(17 ; 53.1 \%)$, with financial difficulties reported by 12 mothers $(37.5 \%)$. Other stressors endorsed were substance abuse by partner $(n=2 ; 6.3 \%)$, physical abuse by partner $(n=3 ; 9.4 \%)$, and physical health problems experienced by the participant $(n=2 ; 6.3 \%)$. Although the majority of mothers were in a significant relationship, more than half reported experiencing a lack of partner support as their main stressor.

\section{Post-partum: Separation}

The majority of mothers $(n=23 ; 71.9 \%)$ had experienced a form of separation from their infants and just over half $(n=18 ; 56.3 \%)$ were separated for a period of a week or more. The reasons for separation included medical procedures $(n=8 ; 34.8 \%)$, maternal psychiatric admission $(n=16 ; 69.6 \%)$, and infants visiting or staying with family members $(n=4 ; 17.4 \%)$. Five mothers $(15.6 \%)$ returned to work, but these were not classified as separations. Separations ranged from the time of birth to 8 months postpartum and the length of separation ranged from 1 day to 9 months, with an average time of separation of 54 days. Eight mothers (36.4\%) experienced multiple separations from their infants during the first 12 months postpartum. Timing of and reasons for separation are summarized in Table 4. 
Table 4

Early separation between mother and infant

\begin{tabular}{|l|lll|}
\hline Time of separation & $\begin{array}{l}\text { \% }(\mathbf{n}= \\
\mathbf{2 3})\end{array}$ & $\begin{array}{l}\text { Length of separation } \\
\text { in days }\end{array}$ & $\begin{array}{l}\text { Average length of } \\
\text { separation in days }\end{array}$ \\
\hline After birth & $\begin{array}{l}30.4 \\
(7)\end{array}$ & 1 to 72 & $16.0(\mathrm{sd}=23.4)$ \\
\hline Within 3 months postpartum & $\begin{array}{l}39.1 \\
(9)\end{array}$ & 2 to 279 & $70.6(\mathrm{sd}=95.7)$ \\
\hline 3 to 6 months postpartum & $\begin{array}{l}30.4 \\
(7)\end{array}$ & 2 to 107 & $56.1(\mathrm{sd}=31.8)$ \\
\hline 6 to 12 months postpartum & $\begin{array}{l}17.4 \\
(4)\end{array}$ & 14 to 76 & $40.5(\mathrm{sd}=22.9)$ \\
\hline Unknown & $\begin{array}{l}8.7(2) \\
\text { Reasons for separation }\end{array}$ & 2 & $2(\mathrm{sd}=0.0)$ \\
\hline Psychiatric admission of mother & $\begin{array}{l}69.6 \\
(16)\end{array}$ & 14 to 107 & $47.6(\mathrm{sd}=26.4)$ \\
\hline Medical reasons & $\begin{array}{l}36.8 \\
(8)\end{array}$ & 1 to 14 & $5.9(\mathrm{sd}=4.8)$ \\
\hline $\begin{array}{l}\text { Child visiting or staying with } \\
\text { family members }\end{array}$ & $\begin{array}{l}17.3 \\
(4)\end{array}$ & 2 to 279 & $123.8(\mathrm{sd}=124.0)$ \\
\hline * 8 dyads experienced more than one separation & \\
\hline
\end{tabular}

The majority of mothers $(n=25 ; 78.1 \%)$ identified themselves as the primary caregiver of their infants and a family member assumed primary responsibility for the infants in the remaining cases. Four mothers did not live with their infants full-time, one due to work commitments and three due to arrangements made following their psychiatric admissions.

\section{Discussion}

This study aimed to describe the socio-demographic factors and pregnancy outcomes for mothers who experienced peripartum psychosis, in particular early separation from their infants, in order to understand the impact of psychotic experiences on mothers' experience of parenthood. This group of mothers experienced several factors that may have contributed to their vulnerability, including obstetric and birth complications, separations from their infants, emergence and/or relapse of symptoms, high rates of trauma, substance abuse and significant psychosocial stressors.

The rate of unplanned pregnancy (87.5\%) in this group of mothers with peripartum psychosis is higher than the $47.2 \%$ rate of unplanned pregnancy in mothers with mental illness examined by Du Toit and colleagues (32) and that of a group of mothers attending Midwife Obstetric Units (MOUs) in Cape Town 
(68.2\%) (16). The higher rate of unplanned pregnancy could be a consequence of the nature of their psychiatric illnesses. Similar to previous studies of mothers with mental illness, the majority of mothers who took part in this study experienced psychiatric and obstetric problems during their pregnancy and delivery $(9,11)$. However, the present study focused on peripartum psychosis, which comprises a significantly smaller portion of the population of mothers with mental illness than those described in Hoirisch-Clapauch et al. (9) and Jablensky et al. (11).

Prenatal substance use was common (50.0\%), with the use of tobacco being the most commonly used substance. Prenatal nicotine use is associated with adverse pregnancy outcomes and negatively impacts child health, behavior and development (13). The rate of smoking (40.6\%) and alcohol use $(21.7 \%)$ reported, were slightly higher than what Vythilingum and colleagues (15) found among women presenting for antenatal visits at a MOU in Cape Town (smoking in 36.8\%, and alcohol use in $20.2 \%$ of pregnant mothers). The self-reported prevalence of alcohol use was lower than the $36.9 \%$ found by Williams and colleagues (16) among pregnant women attending MOUs in Cape Town. The prevalence of alcohol use is cause for concern, as it contributes to the development of fetal alcohol spectrum disorders (FASD) and other long-term negative child outcomes (13). Illicit substance use was reported by $12.5 \%$ of women in this study, which exceeded the rates of $4 \%$ and $3.6 \%$ found by Vythilingum and colleagues (15) and Williams and colleagues (16), respectively. Similar to findings reported by Zhao, McCauley and Sheeran (14), the presence of both maternal mental illness and substance abuse may have contributed to the frequency of obstetric problems and poor birth outcomes seen in this study.

Socio-economic and psychological stressors were prevalent among this group of women, including lack of partner support, financial difficulties, histories of trauma and abuse. These factors may have contributed to the fact that $53.1 \%$ of mothers experienced an emergence or relapse of symptoms that necessitated psychiatric admission, which corresponds with findings reported by Kendell and colleagues (3).

As our study demonstrated, a significant percentage of the mother-infant dyads experienced periods of early separation (71.9\%). Most of these separations were as a result of psychiatric admissions and occurred during the first three months postpartum. Separations that occur during this early period are particularly concerning, as infants rely on the mother's physical proximity as the primary indicator of her availability, which is necessary for the development of secure mother-infant attachment (28). Our study found a higher rate of separation than Cès and colleagues (23), who reported that $27.2 \%$ of mothers with psychotic disorders were separated from their infants following joint admission to mother-baby units reported. As in this study, a large proportion of mothers in the study by Cès and colleagues smoked during pregnancy ( $42 \%$ vs. $40.6 \%$ ), but fewer mothers in their study used alcohol (7.2\% vs. $21.9 \%)$. During the joint admissions reported by Cès and colleagues, mother-infant interaction could be observed: $4.4 \%$ of mothers displayed abusive behavior, while more than half displayed neglect (51.7\%). During pregnancy, $56.0 \%$ of women in their study experienced decompensation, a figure similar to the $50.0 \%$ of mothers in this study who experienced psychotic symptoms. Factors similar to those identified by Cès and colleagues contributed to our group of mothers' early separation from their infants, including maternal 
psychiatric relapse during pregnancy, infant health, and characteristics of mothers' environmental support and social history (23). Although the separations in our study were not due to social service intervention, the potential infant risks for neglect or maltreatment remain.

The National Institute for Health and Care Excellence (NICE) recognizes that women with mental illness may experience difficulties within the mother-infant relationship and recommends admission to a specialized mother-and-baby unit in the event that the mother requires inpatient treatment within the first year postpartum (31). Wisner and colleagues (42) have cautioned that separations between mothers and infants due to mother-only psychiatric admissions pose several dilemmas, including refusal of and longer periods of admission, undermining of breastfeeding, and transfer of responsibility of caring for the infant to spouses and extended family. The current absence of public sector mother-baby units in the Western Cape region of South Africa, where some of the risks to mother-infant interaction and attachment could be observed and potentially mitigated, is of particular concern in this group of women who experienced frequent early separations and multiple psychosocial stressors in addition to their mental illness.

\section{Implications for healthcare}

In combination, the risk factors described in this study highlight the challenges facing a group of women who, as a consequence of their psychiatric illness, may experience challenges in fulfilling their parental role. Mothers with SMI, particularly those with non-affective psychoses frequently experience potentially modifiable risk factors that may jeopardize their pregnancy outcomes (5), similar to those of the population studied in the present sample, such as substance abuse and relational difficulties. Concerningly high rates of substance abuse during pregnancy highlight the need for routine screening and psychoeducation at primary care level, implementation of preventative interventions, with close follow-up and if needed, targeted substance abuse interventions within this population. In addition to identifying the psycho-social risk factors during pregnancy, these women may benefit from close obstetric and psychiatric follow-up during their pregnancy and beyond. As there appears to be an increased risk for post-natal relapse and admissions, there may also be a need for more frequent psychiatric follow-up for early detection of signs of relapse. An integrated care strategy could provide tailored psycho-social support of the dyad to assist these mothers in developing healthy attachments to their infants in light of the frequent early separations experienced. These interventions may be in the form of home visits to the dyad, referral to parent-infant clinics for outpatient support, the provision of parenting support or mother-infant psychotherapy that support the attachment relationship. Future pregnancies carry a significant risk for post-partum psychosis (43), and women may benefit from specific counselling regarding reproductive risks and subsequent vulnerability to illness.

The establishment of Mother and baby units within the South African public health sector would provide an opportunity to provide post-natal psychiatric and psychological care for this group of women who may otherwise be at high risk of developing insecure or disorganized attachments with their infants. These units could provide specialist care and interventions to these vulnerable dyads, which would limit the need for and length of separation between mother and infant within the early postpartum period.

Page 13/19 
Given the elevated risks associated with pregnancy and delivery for this group of women, close psychiatric and obstetric follow-up and targeted substance abuse interventions may improve outcomes experienced by both mother and infant.

\section{Limitations}

The generalizability of this data is limited due to the small sample size and heterogenous nature of the sample. The sample size was comparable to other observational studies of mothers with serious mental illnesses in the postpartum period (44-46), as these patients were relatively scarce. Information on pregnancy and obstetric complications, substance abuse and stressful life events was obtained from patient self-reports. The information may have been more reliable if obtained through obstetric records and structured assessment tools, such as the Life Events Checklist for DSM-5 (LEC-5). As there is currently no long-term follow-up information available, the implications for infant outcomes is unclear. However, the high rate of complications reported may have impacted negatively on the mothers' experiences of pregnancy and delivery, and may have influenced their early experiences of their infants.

\section{Conclusion}

The group of mothers who experienced peripartum psychosis in this study are faced with several challenges, including early separation from their infants, high rates of peripartum psychiatric relapse and admission, substance abuse difficulties, socio-economic risk factors and widespread trauma histories. All these factors impact on the caregiving environment and may compromise the mothers' ability to provide sensitive and nurturing care. This is a sample of women at high risk who would benefit from close followup by a multi-disciplinary team able to assist with a comprehensive bio-psychosocial care approach to the mother-infant dyad.

\section{Abbreviations}

FASD fetal alcohol spectrum disorders

MBU mother-baby unit

MOU midwife-obstetric unit

NICE National Institute for Health and Clinical Excellence

PANSS Positive and Negative Symptoms Scale

SCI-PANSS Structured Clinical Interview for the Positive and Negative Symptoms Scale

SMI severe mental disorders

WHO World Health Organization 


\section{Declarations}

\section{Ethics approval and consent to participate}

Permission to conduct the study and ethical approval for the study was obtained in accordance to the provisions and approval of the Health Research Ethics Committee of Stellenbosch University (S16/01/014). Consent for participation in the study was obtained from the participants. Participation in the study was voluntary and the participants could withdraw at any point without coercion or any negative consequences.

\section{Consent for publication}

Not applicable

\section{Availability of data and materials}

The data that support the findings of this study are available from Stellenbosch University, but restrictions apply to the availability of these data, which were used under license for the current study, and so are not publicly available. Data are, however available from the authors upon reasonable request and with permission of Stellenbosch University.

\section{Competing interests}

The authors declare that they have no competing interests

\section{Funding}

This research was supported by grants from the South African National Research Foundation (TTK180502325336) and the Harry Crossley Foundation.

\section{Authors' contributions}

JV conducted the study and drafted the manuscript. DJHN performed the statistical analysis. All authors read and approved the final manuscript.

\section{Acknowledgements}


The authors wish to thank the mothers who participated in the study. We appreciate the assistance of Karis Moxley for research support.

\section{References}

1. O'Hara MW, Wisner KL. Perinatal mental illness: Definition, description and aetiology. Best Pract Res Clin Obstet Gynaecol. 2014;28(1):3-12.

2. Jones I, Chandra PS, Dazzan P, Howard LM. Bipolar disorder, affective psychosis, and schizophrenia in pregnancy and the post-partum period. Lancet [Internet]. 2014 Nov [cited 2019 Feb 16];384(9956):1789-99. Available from: https://linkinghub.elsevier.com/retrieve/pii/S0140673614612782

3. Kendell RE, Chalmers JC, Platz C. Epidemiology of puerperal psychoses. Br J Psychiatry. 1987;150(5):662-73.

4. Howard LM. Fertility and pregnancy in women with psychotic disorders. Eur J Obstet Gynecol Reprod Biol [Internet]. 2005 Mar [cited 2019 Feb 16];119(1):3-10. Available from: https://linkinghub.elsevier.com/retrieve/pii/S0301211504003641

5. Taylor CL, Stewart R, Ogden J, Broadbent M, Pasupathy D, Howard LM. The characteristics and health needs of pregnant women with schizophrenia compared with bipolar disorder and affective psychoses. BMC Psychiatry. 2015;

6. Doucet S, Jones I, Letourneau N, Dennis CL, Blackmore ER. Interventions for the prevention and treatment of postpartum psychosis: A systematic review. Arch Womens Ment Health. 2011;14(2):8998.

7. Taylor CL, Broadbent M, Khondoker M, Stewart RJ, Howard LM. Predictors of severe relapse in pregnant women with psychotic or bipolar disorders. J Psychiatr Res [Internet]. 2018 Sep 1;104(June):100-7. Available from: https://doi.org/10.1016/j.jpsychires.2018.06.019

8. Taylor CL, Stewart RJ, Howard LM. Relapse in the first three months postpartum in women with history of serious mental illness. Schizophr Res [Internet]. 2019 Feb 1;204:46-54. Available from: https://doi.org/10.1016/j.schres.2018.07.037

9. Hoirisch-Clapauch S, Brenner B, Nardi AE. Adverse obstetric and neonatal outcomes in women with mental disorders. Thromb Res. 2015;135(Suppl. 1):S60-3.

10. Hoirisch-Clapauch S, Nardi AE. Markers of low activity of tissue plasminogen activator/plasmin are prevalent in schizophrenia patients. Schizophr Res [Internet]. 2014;159(1):118-23. Available from: http://dx.doi.org/10.1016/j.schres.2014.08.011 
11. Jablensky A V, Morgan V, Stephen Z, Bower C, Yellachich L-A. Pregnancy, Delivery, and Neonatal Complications in a Population Cohort of Women With Schizophrenia and Major Affective Disorders. Am J Psychiatry [Internet]. 2005 [cited 2019 Mar 14];162:79-91. Available from: http://ajp.psychiatryonline.org

12. Maccabe JH, Martinsson L, Lichtenstein P, Nilsson E, Cnattingius S, Murray RM, et al. Adverse pregnancy outcomes in mothers with affective psychosis. Bipolar Disord. 2007;9:305-9.

13. Louw K-A. Substance use in pregnancy: The medical challenge. Obstet Med [Internet]. 2018;11(2):54-66. Available from: https://doi.org/10.1177/1753495X17750299

14. Zhao L, McCauley K, Sheeran L. The interaction of pregnancy, substance use and mental illness on birthing outcomes in Australia. Midwifery [Internet]. 2017;54:81-8. Available from: http://dx.doi.org/10.1016/j.midw.2017.08.007

15. Vythilingum B, Roos A, Faure SC, Geerts L, Stein DJ. Risk factors for substance use in pregnant women in South Africa. South African Med J [Internet]. 2012 Sep 14;102(11):851. Available from: http://www.samj.org.za/index.php/samj/article/view/5019

16. Williams PP, Jordaan E, Mathews C, Lombard C, Parry CDH. Alcohol and Other Drug Use during Pregnancy among Women Attending Midwife Obstetric Units in the Cape Metropole, South Africa. Adv Prev Med. 2014;2014:1-10.

17. Meltzer-Brody S, Howard LM, Bergink V, Vigod S, Jones I, Munk-Olsen T, et al. Postpartum psychiatric disorders. Nat Rev Dis Prim [Internet]. 2018 Apr 26;4:1-18. Available from: http://dx.doi.org/10.1038/nrdp.2018.22

18. Bergink V, Rasgon N, Wisner KL. Postpartum psychosis: Madness, mania, and melancholia in motherhood. Am J Psychiatry. 2016 Dec 1;173(12):1179-88.

19. Jones I, Smith S. Puerperal psychosis: identifying and caring for women at risk. Adv Psychiatr Treat. 2009;15:411-8.

20. VanderKruik R, Barreix M, Chou D, Allen T, Say L, Cohen LS. The global prevalence of postpartum psychosis: a systematic review. BMC Psychiatry [Internet]. 2017 Dec 28;17(1):272. Available from: http://bmcpsychiatry.biomedcentral.com/articles/10.1186/s12888-017-1427-7

21. Jones I, Craddock N. Bipolar disorder and childbirth: The importance of recognising risk. $\mathrm{Br} \mathrm{J}$ Psychiatry. 2005;186:453-4.

22. Miller LJ, Finnerty M. Sexuality, pregnancy, and childrearing among women with schizophreniaspectrum disorders. Psychiatr Serv. 1996;47:502-5.

23. Cès A, Falissard B, Glangeaud-Freudenthal NMCC, Sutter-Dallay A-LL, Gressier F. Pregnancy in women with psychotic disorders: risk factors associated with mother-baby separation. Arch Womens Ment Health. 2018 May 4;21(6):699-706.

24. Howard LM. The separation of mothers and babies in the treatment of postpartum psychotic disorders in Britain 1900-1960. Arch Womens Ment Health. 2000;3(1):1-5.

25. Bowlby J. The effect of separation from the mother in early life. Ir J Med Sci [Internet]. 1954 [cited 2019 Oct 17];29(3):121-6. Available from: 
http://www.springerlink.com/index/N5P700514R538740.pdf

26. Spitz RA. Hospitalism: An inquiry into the genesis of psychiatric conditions in early childhood. Psychoanal Study Child [Internet]. 1945 Jan 13 [cited 2019 Oct 17];1(1):53-74. Available from: https://www.tandfonline.com/doi/full/10.1080/00797308.1945.11823126

27. Konishi A, So R, Yoshimura B. Mother-infant separation among mothers with mental illness: An exploratory observational study in Japan. Asian J Psychiatr [Internet]. 2018;32:1-4. Available from: https://doi.org/10.1016/j.ajp.2017.11.024

28. Howard K, Martin A, Berlin LJ, Brooks-Gunn J. Early mother-child separation, parenting, and child well-being in Early Head Start families. Attach Hum Dev. 2011;13(1):5-26.

29. Connellan K, Bartholomaeus C, Due C, Riggs DW. A systematic review of research on psychiatric mother-baby units. Arch Womens Ment Health. 2017;20(3):373-88.

30. Baker AA, Morison M, Game JA, Thorpe JG. Admitting Schizophrenic Mothers With Their Babies. Lancet. 1961;278(7201):237-9.

31. National Institute for Health and Clinical Excellence (NICE). Antenatal and Postnatal Mental Health: the NICE Guideline on Clinical Management. NICE Clin Guidel [Internet]. 2014;1-919. Available from: http://www.nice.org.uk/guidance/index.jsp? action=bypublichealth\&PUBLICHEALTH=Mental+health\#/search/?reload

32. Du Toit E, Jordaan E, Niehaus D, Koen L, Leppanen J. Risk factors for unplanned pregnancy in women with mental illness living in a developing country. Arch Womens Ment Health. 2018;21(3):323-31.

33. Hauck Y, Nguyen T, Frayne J, Garefalakis M, Rock D. Sexual and Reproductive Health Trends Among Women With Enduring Mental Illness: A Survey of Western Australian Community Mental Health Services. Health Care Women Int. 2015;36(4):499-510.

34. Suchman NE, Ordway MR, de las Heras L, Mcmahon TJ. Mothering from the Inside Out: results of a pilot study testing a mentalization-based therapy for mothers enrolled in mental health services. Attach Hum Dev [Internet]. 2016;18(6):596-617. Available from: http://dx.doi.org/10.1080/14616734.2016.1226371

35. Wan MW, Green J. The impact of maternal psychopathology on child-mother attachment. Arch Womens Ment Health. 2009;12(3):123-34.

36. Fonagy P, Sleed M, Baradon T. Randomized Controlled Trial of Parent-Infant Psychotherapy for Parents With Mental Health Problems and Young Infants. Infant Ment Health J. 2016;37(2):97-114.

37. World Health Organization, United Nations Children's Fund, World Bank Group. Nurturing care for early childhood development: a framework for helping children survive and thrive to transform health and human potential. Geneva: World Health Organization; 2018.

38. Britto PR, Lye SJ, Proulx K, Yousafzai AK, Matthews SG, Vaivada T, et al. Nurturing care: promoting early childhood development. Lancet. 2017;389(10064):91-102.

39. Zabow T. Competence and decision-making: Ethics and clinical psychiatric practice. South African J Bioeth Law. 2008;1(2):61-3. 
40. Kay SR, Fiszbein A, Opler LA. The Positive and Negative Syndrome Scale (PANSS) for Schizophrenia. Schizophr Bull. 1987;13(2):261-76.

41. Luckhoff H, Phahladira L, Scheffler F, Asmal L, du Plessis S, Chiliza B, et al. Weight gain and metabolic change as predictors of symptom improvement in first-episode schizophrenia spectrum disorder patients treated over 12 months. Schizophr Res. 2019;206:171-6.

42. Wisner KL, Jennings KD, Conley B. Clinical Dilemmas Due to the Lack of Inpatient Mother-Baby Units. Int J Psychiatry Med [Internet]. 1996 Dec 1 [cited 2019 Mar 15];26(4):479-93. Available from: http://journals.sagepub.com/doi/10.2190/NFJK-A4V7-CXUU-AM89

43. Blackmore ER, Rubinow DR, O'Connor TG, Liu X, Tang W, Craddock N, et al. Reproductive outcomes and risk of subsequent illness in women diagnosed with postpartum psychosis. Bipolar Disord. 2013;15(4).

44. Pawlby S, Fernyhough C, Meins E, Pariante CM, Seneviratne G, Bentall RP. Mind-mindedness and maternal responsiveness in infant-mother interactions in mothers with severe mental illness. Psychol Med. 2010;40(11):1861-9.

45. Rigby J, Conroy S, Miele-Norton M, Pawlby S, Happé F. Theory of mind as a predictor of maternal sensitivity in women with severe mental illness. Psychol Med. 2016;46(9):1853-63.

46. Wan MW, Warren K, Salmon MP, Abel KM. Patterns of maternal responding in postpartum mothers with schizophrenia. Infant Behav Dev. 2008;31:532-8.

47. Albertsson-Karlgren U, Graff M, Nettelbladt P. Mental disease postpartum and parent-infant interaction-evaluation of videotaped sessions. Child Abus Rev. 2001;10(1):5-17.

\section{Table 3}

Table 3. Use of substances during pregnancy

\begin{tabular}{ll}
\hline Substance & $\%(n=32)$ \\
\hline Smoking & $40.6(13)$ \\
Alcohol & $21.9(7)$ \\
\hline Illicit drugs & $12.5(4)$ \\
\hline
\end{tabular}

